\title{
EchoGéo
}

19 | 2012

Du littoral à la haute mer : quelles recherches récentes en géographie?

\section{Les géographes et les métiers du tourisme}

Maria Gravari-Barbas et Sébastien Jacquot

\section{(2) OpenEdition}

Journals

Édition électronique

URL : https://journals.openedition.org/echogeo/13009

DOI : 10.4000/echogeo.13009

ISSN : 1963-1197

Éditeur

Pôle de recherche pour l'organisation et la diffusion de l'information géographique (CNRS UMR 8586)

Référence électronique

Maria Gravari-Barbas et Sébastien Jacquot, "Les géographes et les métiers du tourisme », EchoGéo

[En ligne], 19 | 2012, mis en ligne le 10 février 2012, consulté le 28 juin 2022. URL : http://

journals.openedition.org/echogeo/13009; DOI : https://doi.org/10.4000/echogeo.13009

Ce document a été généré automatiquement le 10 août 2021.

EchoGéo est mis à disposition selon les termes de la licence Creative Commons Attribution - Pas d'Utilisation Commerciale - Pas de Modification 4.0 International (CC BY-NC-ND) 


\title{
Les géographes et les métiers du tourisme
}

\author{
Maria Gravari-Barbas et Sébastien Jacquot
}

\section{Géographie et tourisme, une relation ancienne, à faire et à refaire}

1 Le tourisme entretient une relation forte, ancienne et privilégiée avec la géographie française. En France c'est effectivement la géographie qui a dominé, du moins jusqu'à récemment, les études du tourisme. Sa prééminence est désormais disputée par l'économie, l'anthropologie et à un moindre degré la sociologie, mais l'analyse des thèses de doctorat soutenues au cours des trois dernières décennies dans les universités françaises (y compris par des étudiants étrangers) met en évidence la place importante qu'occupe la géographie dans les études du tourisme en France ${ }^{1}$. En revanche, dans les pays anglo-saxons, la discipline géographique est moins prégnante (Holden, 2005) dans l'étude du tourisme.

2 L'importance des géographes n'est pas seulement due au fait que le tourisme est une activité à très forte dimension spatiale, mais aussi aux fondements historiques de la géographie en France. A titre d'exemple, Adolphe Joanne a embauché pour la rédaction de ses guides, ancêtres du Guide bleu, Elisée et Elie Reclus, et plusieurs autres géographes ont collaboré à la rédaction de guides de tourisme (Bruston, 2000). Plus tard, et dans un registre légèrement différent, le texte de Maurice Le Lannou «Itinéraires de Bretagne, guide géographique et touristique » (Le Lannou, 1938), est un exemple pertinent de la proximité, voire de la complicité, entre la plume des géographes et le tourisme, qui s'est poursuivie par des contributions dans les guides bleus (Chevalier, 1997), ou la collection Découvrir la France, coordonnée par Roger Brunet entre 1972 et 1974 aux éditions Larousse, regroupant plusieurs géographes.

Ce n'est pas pour autant que la géographie s'est emparée du tourisme dès l'émergence de ce phénomène, qui depuis son apparition a étendu l'œkoumène touristique. Au contraire, on observe un décalage certain entre le développement et l'affirmation du 
tourisme au XIX ${ }^{e}$ siècle, et son étude par les géographes. A posteriori, on ne peut qu'être étonné qu'un ensemble de faits touristiques spatialement saillants (la station balnéaire ou de montagne, les parcs d'attraction, les routes pittoresques, les expositions internationales générant des flux urbains inédits, etc.) n'ait que très peu attiré l'attention des géographes de la fin du XIX ${ }^{e}$ siècle et de la première moitié du XXe.

Il est symptomatique que cette difficulté de la géographie à se saisir du tourisme ne concerne pas uniquement le tourisme mais aussi, de manière plus générale, la ville destination du Grand Tour dès le XVII ${ }^{e}$ siècle - à laquelle peu de travaux avaient été consacrés avant les manuels de Georges Chabot $(1948)^{2}$ et de Pierre Georges (1952). De manière plus générale le tourisme pâtit aussi de la difficulté qu'éprouvent les géographes jusqu'à une certaine époque à cerner la mobilité - a fortiori, dans le cas du tourisme, une mobilité perçue comme frivole.

5 L'investissement du tourisme par la géographie se fera donc progressivement, mais avec un développement certainement plus affirmé depuis la fin des dernières décennies du XXe siècle. Butler (2004, cité in Williams, 2009), distingue 3 phases successives dans la manière dont le tourisme a été approché par les géographes, tant en ce qui concerne les objets considérés que les méthodologies employées :

- «L'ère de la description », jusqu'au années 1950, caractérisée par un intérêt modéré de la discipline pour le tourisme. Les travaux, essentiellement descriptifs, concernent des lieux considérés a priori commetouristiques : côtes, stations, resorts...

- "L'ère thématique » (entre 1950 et 1980), pendant laquelle les géographes abordent des questionnements tels que les effets d'échelle, la distribution spatiale des phénomènes touristiques et du flux touristique, les relations entre les populations touristiques et les lieux visités, l'impact touristique, la modélisation spatiale du développement touristique. Fortement positivistes, ces approches sont marquées en France par le régionalisme et tendent à aboutir à des typologies parfois rigides qui ont caractérisé pendant longtemps l'étude géographique du tourisme.

- "L'ère de la diversité » finalement, qui marque la dernière décennie du XX $\mathrm{X}^{\mathrm{e}}$ siècle et le début $\mathrm{du} \mathrm{XXI}{ }^{\mathrm{e}}$, cherche à suivre le développement contemporain pluriforme du tourisme, qui s'affirme comme un phénomène social complexe et transversal, touchant, fût-ce de manière inégale ou différenciée, tous les peuples et toutes les sociétés. Les études géographiques se font la caisse de résonance d'un tourisme devenu désormais " genre commun » (Lussault, 2007) et construisent de nouvelles passerelles avec des disciplines comme l'anthropologie, la sociologie, la gestion, l'économie. Elles s'intéressent à la fois à la « production » du tourisme (les acteurs, le rapport entre tourisme et aménagement, les politiques touristiques dans les économies capitalistes, dans les contextes post-coloniaux ou dans les pays émergents, le marketing et le «branding » touristique), à la « consommation » du tourisme (les pratiques touristiques et leur spatialisation, le rapport aux communautés locales, la corporéité (Crouch, Desforges, 2003), les pratiques différenciées sexuées, de classe, ethniques), voire de plus en plus à la « prosumption », néologisme mettant en évidence la relation étroite entre « production » et « consumption» spatiales.

Un champ d'études large s'ouvre ainsi aux études du tourisme, désormais saisi comme un phénomène social total, et plus seulement produit. 


\section{Le tourisme, une réalité économique à contours géographiques}

7 Le tourisme, au niveau international, constitue une activité économique en forte croissance. Ainsi en 2011, les arrivées de touristes internationaux atteignent 980 millions, en croissance de $4 \%^{3}$. En France, la consommation touristique intérieure (consommation des résidents et non résidents) représente 6,2\% du PIB en 2007, et les activités caractéristiques du tourisme représentent 840000 salariés fin $2007^{4}$. Ces données permettent d'appréhender l'importance économique du tourisme, mais surtout laissent entrevoir l'ampleur des métiers du tourisme, qui concernent tant l'hébergement (hôtels, campings, ...), la restauration, que les agences de voyage, la gestion des équipements touristiques (remontées mécaniques par exemple), les cabinets conseil ou les missions tourisme au sein des collectivités (ces deux derniers exemples ne sont pas intégrés dans les statistiques de l'emploi du tourisme, aboutissant à sa sous-évaluation).

Cette diversité impose aussi de revenir à des définitions du tourisme ${ }^{5}$.

9 En 2000, l'OMT et la Commission de statistique de l'Organisation des Nations Unies ont défini le tourisme comme étant « les activités déployées par les personnes au cours de leurs voyages et de leurs séjours dans les lieux situés en dehors de leur environnement habituel pour une période consécutive qui ne dépasse pas une année, à des fins de loisirs, pour affaires et autres motifs non liés à l'exercice d'une activité rémunérée dans le lieu visité ».

10 Les métiers du tourisme relèvent alors de l'ensemble de ces activités, ce qui peut conduire à une approche restreinte de ceux-ci aux métiers de l'hébergement, de l'aménagement touristique, ou relatifs à la gestion des équipements touristiques par exemple. Mais on peut adopter une approche plus large, en considérant que le tourisme est une activité transversale, qui ne peut être cantonnée à des resorts bien délimités, et qui infuse tout un ensemble de territoires. Cette approche remet en cause certaines catégories établies (notamment entre touristes et habitants). Dans une telle approche, des services ordinaires, tels les transports publics ${ }^{6}$, les activités commerciales, etc. revêtent une dimension touristique, et peuvent être intégrés dans des stratégies de développement touristique.

11 Cette dimension transversale apparaît dans la façon dont la géographie française s'est saisie de l'objet « tourisme ». Ainsi G. Cazes appelait à définir le tourisme en prenant en compte tant les touristes que les territoires et impacts. L'équipe MIT (2003) propose une définition systémique du tourisme, le présentant comme "un système d'acteurs, de pratiques et d'espaces qui participent de la "recréation» des individus par le déplacement temporaire hors des lieux du quotidien ".

12 Ainsi, que l'on évoque une géographie du tourisme ou une approche géographique du tourisme (Knafou, Bruston et al., 1997), il s'agit bien de faire droit à la complexité du phénomène, qui peut conduire aussi bien à une analyse des transformations des espaces, à travers par exemple les études de trajectoires de lieux (Equipe MIT, 2005), l'identification des nouveaux territoires du tourisme telles les périphéries urbaines (Maitland, Newman, 2008), ou l'études des aménagements touristiques (Knafou, 1978), qu'à l'analyse des pratiques et expériences touristiques ${ }^{7}$. Les études sur le tourisme s'opèrent également au croisement d'autres enjeux, par exemple la patrimonialisation 
(Gravari-Barbas, 2005 ; Lazarotti, 2011), l'environnement (Deprest, 1997), ou les dynamiques événementielles.

Des notions endogènes à la pratique professionnelle du tourisme révèlent également la dimension spatiale du tourisme. Ainsi, la notion de destination touristique, qui n'est pas une notion issue de la géographie mais qui relève de la terminologie des acteurs du tourisme, constitue une notion éminemment spatiale, qui pose des questions aux géographes. Désignant l'espace perçu comme cohérent par des visiteurs, avec un périmètre plus ou moins défini, pouvant transgresser des limites administratives ou fonctionnelles, relevant à la fois des pratiques et des imaginaires, la destination touristique est en même temps un enjeu des politiques publiques et privées du tourisme, tant en terme de communication que d'aménagements. L'intercommunalité par exemple, avec ses stratégies de communication, de mutualisation de moyens, peut se donner pour but la construction de nouveaux territoires touristiques, dont l'existence est légitimée par leur cohérence du point de vue touristique, bref en devenant des destinations. De ce point de vue, la décentralisation a multiplié les politiques territoriales de développement touristique mais aussi les reconfigurations territoriales. Quelle place alors dans ces mouvements peut occuper la science géographique?

\section{Le tourisme, quels débouchés pour les géographes?} nationales tout d'abord, régionales ensuite. A partir des dernières décennies du $\mathrm{XX}^{e}$ siècle, la décentralisation et le "réveil du local » ont été bénéfiques au développement des métiers du tourisme laissant une large place aux géographes. Ceci concerne à la fois le secteur privé, public et associatif.

16 Au cours des dernières décennies du XXe siècle, le tourisme s'est imposé de plus en plus aux territoires comme une des cartes à jouer pour se singulariser. Cette course à la singularisation s'est exprimée aussi bien par le développement de nouvelles «attractions» touristiques (resorts, parcs d'attraction, parcs de loisirs) que par la requalification des traces du passé, monumentales ou modestes. Les touristes ont été des pionniers de ce mouvement et ont eux-mêmes libéré le tourisme des a priori déterministes le limitant aux côtes pittoresques, aux belles plages et aux stations de montagne. Les décideurs locaux des anciennes régions industrielles, les vieux ports, les grandes villes ou les territoires ruraux ont cherché à mettre en évidence les qualités de leurs territoires et de leurs patrimoines. Dans certains territoires c'est la démarche inverse qui prévaut: on fait appel aux compétences géographiques non pas pour développer mais pour modérer les effets d'une fréquentation considérée comme trop importante ou pour intervenir sur la spatialisation des conflits et des tensions entre populations permanentes et de passage, ou entre différentes fonctions dans l'espace.

17 Le secteur public territorial a ainsi été un des principaux pourvoyeurs d'emplois pour les géographes. Le développement des inter territorialités a dynamisé davantage, a prolongé ou a renouvelé ces initiatives. 
Le secteur privé s'est également inséré dans cette dynamique. Un nombre important de cabinets conseil ou d'ingénierie touristique ont été créés au cours des dernières années. Si leurs marchés en France sont encore actifs, c'est désormais vers l'international que tend à se déplacer l'activité conseil.

9 Les savoir-faire géographiques sont ainsi mobilisés en amont du lancement des projets, dans le lancement d'études préalables, de faisabilité, de marché. Les diagnostics territoriaux mobilisent des outils cartographiques d'analyse du territoire, d'analyse des jeux d'acteurs dans lesquels les géographes ont leur mot à dire. En aval, les études d'impact font également largement appel aux compétences géographiques. Que ce soit dans une approche de marketing ou de branding territorial ou dans la création de nouvelles identités locales, le tourisme se conjugue bien avec le bagage géographique.

La dynamique associative a également accompagné le développement des territoires en se faisant aussi la caisse de résonnance de nouvelles préoccupations contemporaines, notamment dans les domaines du patrimoine et de l'environnement.

1 Le développement des préoccupations patrimoniales et environnementales a été accompagné par les universités qui ont été nombreuses à mettre en place des DESS dans un premier temps, des Masters professionnels par la suite, de plus en plus spécialisés, conjuguant le développement du tourisme à l'aménagement des territoires, à la requalification patrimoniale, aux questions environnementales.

22 A un niveau de qualification plus important (Doctoral) la recherche fondamentale ou appliquée conduit également à des métiers liés au tourisme. Si les débouchés universitaires restent limités, malgré leur développement considérable au cours des dernières années, la recherche appliquée pourrait être appelée à se développer encore dans les années à venir. Le dispositif des bourses doctorales CIFRE ${ }^{8}$ concerne ainsi non seulement le secteur public, mais aussi les entreprises en particulier dans le secteur du conseil.

Dans le cadre de ce dossier nous avons sollicité des entretiens avec des professionnels représentant cet ensemble de secteurs: institutionnel, territorial, privé, recherche. Noel Le Scouarnec, de la Direction Générale de la compétitivité, de l'industrie et des services, du Ministère de l'Économie, de l'Industrie, et des Finances, expose un parcours au sein de structures institutionnelles nationales qui articulent la connaissance du tourisme à l'action territoriale et l'aménagement, mobilisant des savoir-faire issus d'un parcours en géographie. Michael Duarte, chargé de développement au Comité départemental du tourisme de Seine-Saint-Denis, insiste sur les apports de la formation en géographie, qui permet de construire des projets de développement touristique sous-tendus par une connaissance fine du territoire et de ses dynamiques. Fabien Hennion, passé par un cabinet d'études, est à présent chargé de mission à l'Union Départementale des Offices de Tourisme et Syndicats d'Initiative du Nord (UDOTSI). Dans le cadre de ses missions, il insiste sur la polyvalence acquise par la formation en géographie, mais aussi l'aptitude à territorialiser, et ainsi construire un pont entre une approche administrative et une approche marketing du tourisme (la destination) : la formation de géographe le conduit à être attentif aux évolutions territoriales et aux enjeux territoriaux des jeux d'acteurs. Enfin, Linda Boukhris est doctorante en géographie, menant une thèse sur l'écotourisme au Costa Rica, à travers ses imaginaires et les politiques menées. Mobilisant les savoirs géographiques issues de sa formation initiale, elle rappelle également la nécessité de les confronter à d'autres corpus 
théoriques issus des sciences sociales. En poste également dans un cabinet conseil, elle relève les apports croisés de ses deux activités, en terme méthodologique notamment.

Les personnes interviewées sont ainsi représentatives des parcours des géographes entre différents secteurs du tourisme. En effet, plusieurs sont passées du privé au public, du territorial à l'institutionnel, du privé à la recherche. La géographie mène certes à tout...mais ces transferts et évolutions témoignent surtout du caractère transversal, pluridimensionnel et, dans le bon sens du terme, généraliste, des métiers géographiques du tourisme.

\section{BIBLIOGRAPHIE}

Bruston M., 2000. Des bains au tourisme, la tentation de l'eau au début de la Troisième République. Mappemonde, 2000, numéro 2.

Chevalier J.-P., 1997.Quatre pôles dans le champ de la géogaphie ? Cybergéo, article 23.

Crouch, Desforges, 2003. The sensuous in the tourist encounter: the power of the body in tourist studies. Tourist studies, vol 3 (1), 5-22

Deprest F., 1997. Enquête sur le tourisme de masse : l'écologie face au territoire. Paris, Belin.

Équipe MIT, 2005. Tourismes 2 : moments de lieu. Paris, Belin.

Gravari-Barbas M. (dir.), 2005. Habiter le patrimoine : enjeux, approches, vécu. Rennes, Presses universitaires de Rennes.

Holden, A., 2005. Tourism studies and the social sciences. London, New York, Routledge.

Knafou R., 1978. Les stations intégrées de sports d'hiver des Alpes françaises : l'aménagement de la montagne à la française. Paris, Masson.

Knafou R., Bruston M., Deprest F., Duhamel P., Gay J.-C., Sacareau I. Une approche géographique du tourisme. L'espace géographique, 1997, n 3.

Lazzarotti, O., 2011. Patrimoine et tourisme. Paris, Belin.

Le Lannou Maurice, 1938. Itinéraires de Bretagne, guide géographique et touristique. Paris, Baillière.

Lussault M., 2007; Le tourisme, un genre commun. In Duhamel, Knafou, Mondes urbains $d u$ tourisme, Paris, Belin, p. 333-349.

Maitland, R., Newman, P., 2008. World tourism cities. New York, Routledge.

Williams Stephens, 2009. Tourism geography, a new synthesis. London, Routledge.

\section{NOTES}

1. Il convient également de rappeler que la situation entre les disciples n'est pas la même. Une thèse en géographie permet encore de faire en France des belles carrières universitaires, ce qui 
n'est pas le cas pour d'autres disciplines telles que l'économie, où le tourisme a encore à gagner ses lettres de noblesse.

2. Il est d'ailleurs révélateur que le premier lieu de formation consacré au tourisme ait été imaginé par un géographe intéressé par la ville. En effet , c'est Georges Chabot qui met les bases d'un premier centre d'études touristiques, qui donnera naissance en 1961, grâce à un autre géographe, Louis Burnet, au CEST (Centre d'Etudes Supérieures du Tourisme), aujourd'hui IREST.

3. OMT, communiqué du 16 janvier 2012.

4. Direction du tourisme, 2008, Les comptes du tourisme 2007.

5. En effet, plusieurs définitions coexistent, toutes ont leurs avantages et défauts. Ainsi celle utilisée par l'OMT permet d'établir des statistiques, mais mêle des phénomènes que certains veulent séparer : mobilités d'affaires, séjours médicaux, pélerinages, etc.

6. Comme le montre la thèse de Gwendal Simon, soutenue en 2010 : Pratiques touristiques dans la métropole parisienne, une analyse des mouvements intra-urbains, et réalisée à l'Université de Paris-Est en CIFRE au sein de la RATP.

7. L'usage de la notion de performativité et performance ont ainsi permis de nouvelles approches, cf. Edensor, 1998, Tourists at the Taj, performance and meaning at a symbolic site, Routledge. Pour une présentation détaillée: Chapuis, 2010, Performances touristiques, d'une métaphore à un cadre géographique renouvelé, Mondes urbains du tourisme, n 2;

8. Le dispositif CIFRE [Conventions Industrielles de Formation par la Recherche] subventionne les entreprises de droit français qui embauchent un doctorant rattaché à un laboratoire public.

\section{AUTEURS}

\section{MARIA GRAVARI-BARBAS}

Maria Gravari-Barbas, Enseignante, est directrice de l'IREST, EIREST, Université Paris 1 Panthéon Sorbonne. Maria.Gravari-Barbas@univ-paris1.fr

\section{SÉBASTIEN JACQUOT}

Sébastien Jacquot est Enseignant à l'IREST, EIREST, Université Paris 1 Panthéon Sorbonne.

Sebastien.Jacquot@univ-paris1.fr 